\title{
Tobacco Consumption and Oral, Pharyngeal and Lung Cancers
}

\author{
Maaly A. Bassiony ${ }^{1,2}$, Madeeha Aqil ${ }^{1}$, Maryam Khalili1 ${ }^{1}$, James A. Radosevich ${ }^{1,3, *}$, \\ Heba M. Elsabaa ${ }^{4}$ \\ ${ }^{I}$ Department of Oral Medicine and Diagnostic Sciences, College of Dentistry, University of Illinois at Chicago, \\ Chicago, IL, 60612, USA \\ ${ }^{2}$ Oral and Maxillofacial Surgery, College of Dentistry, Mansoura University, Egypt \\ ${ }^{3}$ Jesse Brown VAMC, Chicago, IL, 60612, USA \\ ${ }^{4}$ Department of Oral Biology, Faculty of Dentistry, Mansoura University, Egypt
}

\begin{abstract}
Tobacco consumption remains a significant threat to public health around the world and smoking-related diseases are considered the world's most preventable cause of death. Smoking is associated to several diseases and conditions, most importantly different types of cancer. At least $30 \%$ of all cancer death is related to smoking including $87 \%$ and $70 \%$ of lung cancer deaths in men and women, respectively. Lung cancer is the leading cause of cancer deaths compared to cancer of other organs in both men and women. Also, tobacco is a known risk factor of oral and pharyngeal malignancies. Although oral cancer accounts for $1 \%-2 \%$ of all cancers in the body, it carries a relatively high rate of mortality. It has been shown that the risk of oral cancer is reduced by smoking cessation. Also, mortality and morbidity of oral cancer could be effectively reduced by elimination of this risk factor. In this article, we review the current literature on the relationship of tobacco with oral, pharyngeal and lung cancers. We present the epidemiologic and experimental evidence supporting the link between tobacco use and the induction and development of cancer. The association between genetic susceptibility and tobacco carcinogens is discussed. supported by genome-wide association studies. Public health preventive measures and tobacco control means are emphasized as well.
\end{abstract}

Keywords: Tobacco, Smoking, Mouth neoplasm, Lung, Pharynx, Epidemiology, Risk factors, Genetics, Susceptibility.

\section{INTRODUCTION}

Tobacco consumption is a serious global problem and significant health hazard which could be harmful to almost every organ in the body and ultimately leading to death. Each year, 6 million people die from tobacco use and it is estimated that by 2030, the number would rise to 8 million deaths annually. Smoking is directly related to many diseases and conditions including heart disease, cerebrovascular disease, chronic bronchitis, emphysema and gastric ulcers. Smoking increases the risk of malignant tumors in nearly all organs. Also, at least $30 \%$ of all cancer deaths smoking is attributed to smoking. When the topic of tobacco use is considered, lung cancer is usually the primary epidemiologic concern discussed. Lung cancer is the leading cause of cancer deaths compared to other organs and smoking accounts for $87 \%$ of lung cancer deaths in men and $70 \%$ in women [1]. According to a meta-analysis study from 1961 to 2003 on cigarette smoking and cancer conducted to quantify the risk of 13 cancer sites, the highest relative risks were lung, laryngeal and pharyngeal cancers followed by upper digestive tract and oral cancers [2].

*Address correspondence to this author at the Department of Oral Medicine and Diagnostic Sciences, College of Dentistry, University of Illinois at Chicago, Chicago, IL, 60612, USA; Tel: +1-312-996-9538;

Fax: +1-312-996-9592; E-mail: jrados@uic.edu
Oral cavity and oropharynx are also directly exposed to tobacco consumption. It is widely accepted that tobacco use is the major risk factor in the development of oral cancer. Cigarette smoking increases the risk of oral cancer by two to five times and the risk increases with the years and the numbers of cigarettes smoked. The risk of oral cancers could be reduced by smoking cessation, resulting in $50 \%$ reduction in five years. Interestingly, ten years after smoking cessation, the risk of developing oral cancer approaches that of lifelong nonsmokers [3]. Tobacco consumption is not limited to smoking and there are several tobacco-related habits around the world. In addition, another tobacco-related problem is the secondhand smoke. Tobacco consists of many different chemicals and it is crucial to define the carcinogenic potential of its constituents. This review article provides detailed discussion about recent findings in oral, pharyngeal, and lung cancer focusing on the effect of tobacco use.

\section{WORLDWIDE TOBACCO USE}

In many low and middle income countries (and some high income countries), smoking prevalence has increased during the past two decades, while it was slowly declining in the United States. In 2011, tobacco use killed almost 6 million people globally (about $80 \%$ in low and middle income countries). In 2030, tobacco-attributable deaths are expected to decline by $9 \%$ in high-income countries. 
Currently, tobacco use is the number one killer in China, responsible for 1.2 million deaths each year which is anticipated to increase to 3.5 million deaths by 2030. If current trends continue, more than 8 million people will die worldwide because of tobacco use each year by 2030. In 32 countries, male smoking prevalence is greater than or equal to $45 \%$. Female smoking prevalence is also on the rise in many low income and middle income countries. In 2004, it was estimated that more than 600,000 nonsmokers worldwide died as a result of exposure to secondhand smoke. The first global public health treaty, the Framework Convention on Tobacco Control (FCTC), was adopted by the World Health Assembly on May 21, 2003. Parties to the treaty should reinforce national legislation, authorize effective tobacco control policies, and work together internationally to reduce global tobacco consumption. In September 2011, 174 out of 195 eligible countries approved the treaty representing approximately $87 \%$ of the world's population. The major tobacco-producing nations, including Argentina, US, Indonesia, Malawi and Zimbabwe signed but did not ratified the treaty [4].

\section{DIFFERENT TYPES OF TOBACCO CONSUMPTION}

\section{Cigarette Smoking}

Cigarette smoking is the typical form of tobacco consumption. The prevalence of cigarette smoking among adults 18 years of age and older showed significant declined during past decades. In 2011, nearly 41.5 million adults were current smokers, which was 4 million fewer than in 2005. The proportion of daily light or intermittent smokers (less than 10 cigarettes per day) increased significantly between 2005 and 2012, whereas heavy smoking declined from 13\% to $7 \%$. Cigarette smoking was more prevalent among men, but the gender gap narrowed in the mid-1980s and has remained constant thereafter.

Smoking is also related to educational status being most common among the least educated. Also, considering decrease in smoking in all educational levels, college graduates had the greatest decline, from 1983 to 2012.

Cigarette smoking among US high school students increased from 1991 to 1997, and declined by 2012. Smoking prevalence among high school students between the late 1970s and early 1990s declined more rapidly among African Americans than whites. As a consequence, lung cancer among adults younger than 40 years of age, which showed higher rates in African Americans, converged [1].

\section{Cigars}

Health hazards from cigar smoking are similar to cigarette smoking and smokeless tobacco. Cigars have lower tax rates compared to cigarettes, and this could be a reason for some people to choose cigars over cigarettes. The total cigarette consumption declined from 2000 to 2011, however, large cigar consumption (including cigarillos) increased by more than two-fold. In 2011, cigar smoking among US high school students declined compared to 1997 . In $2012,5 \%$ of adults 18 years of age and older were current cigar smokers which includes $9 \%$ of men and $2 \%$ of women. African Americans had the highest prevalence of cigar use, followed by American Indians/Alaska Natives, whites, Hispanics, and Asians. Cigar smoking is associated with an increased risk of many cancers including lung, oral cavity, larynx, esophagus, and probably pancreas and the risk of dying from oral, laryngeal or esophageal cancers is 4 to 10 times higher in cigar smokers compared to nonsmokers [1].

\section{Smokeless Tobacco and Related Products}

There are many forms of smokeless tobacco (SLT) products which include dissolvable nicotine products (Orbs, Strips, and Sticks), moist snuff, chewing tobacco, snus (a "spitless," moist powder tobacco pouch) and a variety of other tobacco-containing products that are not smoked. Tobacco companies are currently marketing for smokeless tobacco products as an alternative in non-smoking areas and as a way to quit smoking. In fact, there is no evidence that these products are effective method for cessation treatments. Therefore, the use of smokeless tobacco products as a harmless substitute to quit smoking is false; these products also cause oral, esophageal, lung, pancreatic and other cancers [4]. Precancerous lesions, gum recession, bone loss around the teeth, and tooth staining are other side effects of SLT in the oral cavity, SLT can also lead to nicotine addiction [1]. The consumption of smokeless tobacco is massively growing globally. The majority of smokeless tobacco products are consumed in South Asia [4]. It should be noted that when different types of SLT tobacco are considered and analyzed in details, controversial findings were reported [5].

In the United States, 8.9 million Americans (3.5\%) used smokeless tobacco with most of the users being located in the South and the Midwest (4.3\% and 3.9\% respectively) compared to the West and the Northeast $3.0 \%$ and $2.1 \%$ respectively) [6]. Whites are more likely to use tobacco products than African Americans, Latinos, or Asians [7].

\section{Secondhand Smoke}

Involuntary exposure to tobacco smoke, secondhand smoke (SHS), or environmental tobacco smoke contains numerous carcinogens. In 2007-2008, more than 88 million nonsmoking Americans 3 years of age and older were exposed to SHS. At least 69 out of 7000 SHS chemicals can cause cancer and SHS is responsible for nearly 3400 deaths from lung cancer in nonsmoking adults annually. Coughing, wheezing, chest tightness, and reduced lung function are associated with SHS in nonsmokers. Public policies should be directed toward protecting people from harmful effects of SHS and the most effective approaches to prevent exposure to SHS are smoking prohibition in public places and creating smoke-free environments. Smoke-free policies also decrease the prevalence of both adult and youth smoking. Currently, smoke-free laws have become quite common and $49 \%$ of the US population is covered by a $100 \%$ smoke-free policy in workplaces, restaurants, and bars [1]. 


\section{Tobacco Carcinogens}

Tobacco is known to have around 4,200 different chemicals. The chemical composition of the plant changes as it grows and also during the preparation of the processed products. Smokeless tobacco contains toxic metals such as mercury, lead, and chromium; smokeless tobacco is known to contain various carcinogenic compounds as well, such as polycyclic aromatic hydrocarbons, lactones, coumarin, ethyl carbamate, some volatile aldehydes, volatile $\mathrm{N}$-nitrosamines, nitrosamino acids, tobacco specific N-nitrosamines, inorganic compounds, radioactive Polonium 210, and Uranium 235 \& 238 [7].

Specifically, (S)-N'-nitrosonornicotine $((S)-\mathrm{NNN})$ has been identified as a strong oral carcinogen to humans. $(S)$ NNN is the enantiomeric counterpart of $(R)-\mathrm{NNN}$ and exhibits a higher tumorigenic potency than $(R)$-NNN. $(S)$ $\mathrm{NNN}$ is also more frequently found than $(R)-\mathrm{NNN}$ (average $62.9 \pm 6.3 \%$ ) in tobacco. The absolute (wet weight) amount of $(S)-N N N$ in moist snuff averaged $1.26 \pm 0.5 \mu \mathrm{g} / \mathrm{g}$ tobacco; $0.70 \pm 0.2 \mu \mathrm{g} / \mathrm{g}$ tobacco in smokeless products; and $1.36 \pm 0.6$ $\mu \mathrm{g} / \mathrm{g}$ tobacco in cigarettes [8].

Researchers at University of Minnesota conducted a study on NNN's relationship to esophageal and oral tumors by administering $(S)$-NNN and/or $(R)$-NNN to four groups of rats. Twenty rats were given $(S)$-NNN with tap water, twenty-four were given $(R)$-NNN with tap water, twelve were given a racemic mixture of both NNN enantiomers with tap water, and twenty-two were just given tap water. The doses given to the rats were approximated according to the amount of $(S)$-NNN a smokeless tobacco user would be exposed to due to chronic use of smokeless tobacco products. They were given these mixtures as their source of drinking water for seventeen to twenty months [9]. This study showed that $(S)$-NNN is highly carcinogenic of the oral cavity in rats (and perhaps humans) and the $(R)-\mathrm{NNN}$ enantiomer is not highly carcinogenic by itself. If there is a racemic mixture of the two NNN enantiomers, they work synergistically and the risk of developing oral or esophageal cancer is amplified. It is known that $(S)$-NNN is the more abundant form of the NNN enantiomers in smokeless tobacco, so it is important to understand how this research corresponds to human smokeless tobacco consumption. It is also known that $(S)$-NNN is one of the main culprits of causing oral cancer. Future studies are needed to calculate how much is ingested by humans and how this amount can be decreased.

\section{Oral and Pharyngeal Cancer}

Smoking does not only negatively affect the lungs, but it negatively affects everything in the upper aero-digestive pathway as well. Oral and pharyngeal cancers are considered a serious and growing problem in many parts of the world and they are ranked among the top ten locations of cancer sites $[1,2]$.

The most common type of oral cancer is squamous cell carcinoma (SCC). Other oral cancer types include malignant salivary gland tumors, sarcomas of soft tissues and jaw bones, melanoma, malignant odontogenic tumors, lymphoreticular malignancies, and metastases from tumors located in other organs [3]. Since more than $90 \%$ of oral cancer cases are SCCs and this type is known to be associated with tobacco use, both terms are used interchangeably in the literature regarding oral cancer.

Oral cancer is seen more frequently in men than in women, and it is usually associated with a low socioeconomic status. Oral cancer is most prevalent in Melanesia, South Central Asia, and Central Europe \& Eastern Europe [10]. In South-East Asia, oral cancer is the second most prevalent form of cancer and the second most frequent cause of cancer death in males. One third of all oral cancer cases worldwide and one half of all oral cancer deaths occur in this region [11].

It has be shown that the use of tobacco products, smokeless or smoked, leads to oral and pharyngeal cancer and will also cause complications during a patient's cancer recovery process if tobacco use is continued. Individuals have a lower risk of developing oral and pharyngeal cancers if they quit using tobacco products compared to those who are current users. The shorter the duration an individual uses a tobacco product and/or the smaller the quantity of tobacco product used will lead to a lower risk of developing oral and pharyngeal cancers. Smoking, smokeless tobacco, and alcohol are all risk factors for developing oral and pharyngeal cancers, but studies have shown that these substances will have synergistic effects and will increase the risk of developing oral and pharyngeal cancers. It is important to make it well known to the public that both forms of tobacco will lead to oral and pharyngeal cancer. Some may believe that even with proper education, smokeless tobacco users will continue to use smokeless tobacco products. A study in India has shown that increased knowledge of smokeless tobacco leading to oral cancer was actually a deterrent to use of smokeless tobacco [12]. Since it was proven to help in India, it is important to educate individuals worldwide who are unaware of the risks of tobacco products to prevent them from starting to use tobacco products or to help them quit using tobacco product.

Oral cancer involves a multifactorial carcinogenic pattern consisting of endogenous (genetic) and exogenous (environmental and behavioral) factors [6].

Tobacco consumption in any form, is the major risk factor for oral cancer and is associated with 90 percent of oral cancers in men and 60 percent in women [13]. Worldwide, $42 \%$ of oral and pharyngeal cancer deaths are due to smoking and in high income countries this figure increases to $70 \%$ [2]. The risk of oral cancer development is two to five times higher in smokers compared to nonsmokers and the risk increases with the years and number of cigarettes smoked [13].

In a meta-analysis study of smoking and cancer it was found that there is differential susceptibility for current smokers and former smokers with higher susceptibility in current smokers due to continuous exposure to the risk factor [2]. 
Another retrospective cohort study included a group of 92 male incident cases (individuals who had oral cancer or pharyngeal cancer) and 230 male controls. Life style history and smoking habits were recorded. The odd ratio was calculated to determine the relative risk of many different cigarette use categories. The risk for oral cancer was calculated based on if the subject was a current smoker (individuals who smoked within 6 months of the interview), ex-smoker (individuals who had quit smoking 6 months prior to the interview, ever smoker (current \& ex-smokers), or non-smoker. They calculated the risk of oral cancer or pharyngeal cancer corresponding to the amount of years spent smoking, to the amount of cigarettes smoked per day, based on the type of tobacco used (blonde or black), and based on if the subjects were non/light drinkers $(<2$ drinks/day) or moderate/heavy drinkers (>2 drinks/day) [14].

Ever smokers have a higher chance of developing oral cancer or pharyngeal cancer compared to someone who has never smoked. This overall risk varies if an individual had quit smoking; if the individual had quit smoking, their odds of having oral cancer or pharyngeal cancer are reduced drastically compared to those who identified themselves as current smokers. Ex-smokers who smoked less than 20 cigarettes a day had a much lower risk of developing oral cancer or pharyngeal cancer compared to those who smoked greater than 20 cigarettes a day. Regardless if they were current smokers or ex-smokers, individuals who smoked for more than 30 years had a higher risk of developing oral cancer or pharyngeal cancer compared to individuals who had smoked for less than 30 years. The study also shows that the risk of developing oral cancer or pharyngeal cancer is lower if an individual smokes blonde tobacco instead of black tobacco [14].

A recent case-control study in New England has shown that use of smokeless tobacco is directly related to a greater risk of oral and pharyngeal cancer and increased use and duration of smokeless tobacco increases the risk of developing these types of cancers. The study also showed that if a person is a non-smoker yet still a smokeless tobacco user, their risk of head and neck squamous cell carcinomas was still evident [15].

The second major risk factor is excessive intake of alcohol. Tobacco and alcohol are independent risk factors for developing oral cancer and they act synergistically [13]. It has been suggested that all forms of smoking (cigarette or cigar) have similar oral cancer risks, but there is no clear evidence regarding specific alcoholic drinks (wine, beer, spirits) [16].

A case-control study in the South Indian state of Tamil Nadu was conducted on a large group of non-smoking, nondrinking adults and their mortality rates with various cancers. The study used retrospective analysis on a group of deceased non-smoking, non-drinking individuals who had cancer as their underlying cause of death. Information about the deceased individuals was obtained by questioning their family members about their smoking, drinking, and chewing habits. The other group in the study was generally healthy (non-smoking, non-drinking) individuals. Researchers also used the controls to run a cross-sectional analysis of the relation between chewing status and education level. The researchers found that there was a higher proportionality of tobacco chewers when compared to controls. They found that the ever chewing cases were associated with a fivefold higher mortality from oral cancer compared to the controls and they also had a 1.5 to twofold higher mortality in upper aerodigestive cancers as well as stomach and cervical cancers. Due to their cross-sectional analysis, they found that tobacco chewing was 5 times more prevalent in men and that there was a direct correlation between lower education levels and use of chewing tobacco. It is expected that the combination of chewing and smoking or chewing and drinking will raise an individual's risk of developing oral cancer along with various other types. This study shows that chewing tobacco on its own is directly related to increased mortality of oral cancer and other upper aerodigestive cancers along with stomach and cervical cancers [13]

The high incidence and mortality rates in South-East Asia is attributed to lifestyle risk factors such as tobacco smoking, betel quid chewing and alcohol drinking. Tobacco use is prevalent in South-East Asia along with different tobacco consumption modalities, such as bidi, kreteks, sulpa,chilum, hookli and waterpipes, which may account for more than one half of the total amount of smoked tobacco. Betel quid/ areca nut chewing is also prevalent and there is a great spectrum of ingredients such as tobacco, spices, sweeteners, lime and catechu and different patterns of consumption. Therefore, oral cancer patients from SouthEast Asia are frequently exposed to one or more of these lifestyle risk factors and, unsurprisingly, oral cancer risk is extremely high in smoking drinking-betel quid chewing individuals. Recently, Petti a al published a meta-analysis to assess the magnitude of the smoking-drinking-chewing interaction effect on oral cancer in south-east Asia. The study showed that the smoking-drinking-betel quid chewing interaction has the power to increase the risk of oral cancer by twenty-three to thirty-four times. This interaction is also responsible for more than two thirds of oral cancer cases occurring in this area [11]. Emerging risk factors have been suggested for the development of oral cancer such as HPV, immunosuppression, diet and nutrition and socio-economic status. Also, there are controversial factors with limited evidence such as ethnicity and race, oral hygiene and dentition and indoor air pollution. There is not enough evidence for association of oral cancer with alcohol in mouthwashes, HIV infection, nicotine replacement therapy and Marijuana (cannabis) smoking [16]

Increased consumption of alcohol and smoking in people of low body mass indices (BMI) can increase the odd ratios (OR) of oral, oropharyngeal, hypopharyngeal, and laryngeal cancers; however, there are no comprehensive sex-specific comparisons of ORs for these factors. The greater the amount of tobacco smoking and alcohol consumption combined with a lower body mass index (BMI) the higher odds ratios. ORs were increased in people who were underweight $(<18.5 \mathrm{BMI})$ relative to people of normal weight (18.5-24.9) and decreased in people who were in the overweight and obese categories ( $\geq 25 \mathrm{BMI}$ ) for all sites and 
were homogeneous by sex. ORs by smoking and drinking were significantly higher in women compared with men for oropharyngeal cancer, evocative for hypopharyngeal cancer, but the same for oral cavity and laryngeal cancers [17].

About 15 to $20 \%$ of oral cancer cases occur in patients without major risk factors. The etiology of the disease in individuals without a history of drinking and/or smoking is unclear. Recently there has been an increase in the number of cases among individuals without history of smoking and/or alcohol consumption and in female with a smaller tumor size and location in non-lingual sites. A viral association has been considered for the development of OSCC for this particular group (tobacco and alcohol non-users), such as papilloma virus (HPV) especially HPV-16 and HPV-18 [6].

Oropharyngeal cancers have been significantly associated with oral HPV infections [18]. Individuals with HPVpositive tumors had a higher survival rate than individuals with HPV-negative tumors (individuals with oropharyngeal tumors not caused by HPV; possibly caused by use of tobacco products) [19].

\section{Tobacco Cessation and Oral Cancer Prevention}

Despite recent improvements in the diagnosis and treatment of oral cancer, the mortality rate is significantly high. Generally, about $50 \%$ of patients with oral cancer present with advanced disease. Therefore, early diagnosis is the key to reduce cancer morbidity and mortality. Even more important is the prevention of oral cancer, which can be achieved mainly by cessation of tobacco and alcohol consumption. Tobacco cessation results in a lower incidence of oral cancer and smoking cessation reduces the risk of oral cancer resulting in 50\% reduction in five years. Interestingly, ten years after cessation, the risk of developing oral cancers approaches that of nonsmokers. Tobacco cessation counseling could be performed by dentists and also by dental auxiliary personnel, such as dental hygienists [3].

Viswanath et al conducted a study to examine whether a higher proportion of current and former smokers reported to have an oral cancer screening (OCS) exam in the past year compared with never smokers. Current and former smokers are considered high-risk groups who need to be targeted for oral cancer exams by dental professionals and primary care providers. The findings showed that while the proportion of never and former smokers who reported receiving an OCS exam in the past year increased. During the study period, the proportion of current smokers who reported having the exam remained fairly steady. In other words, people with the highest risk for developing oral cancer were the least likely to report being screened. When the variable "dental visit in the last year" was included in the adjusted analysis, it became evident that a visit to a dental professional was the main difference in OCS between current and never smokers. Dental visit in the last year was the strongest predictor of OCS [13].

Long-term smokers were less likely to report visiting a dentist in the previous year. The authors concluded that annual dental visits should be encouraged for all persons, especially current and former smokers which hold a higher risk for developing oral cancers. Dental health providers should also consider the opportunity to screen for oral cancer at any office visit and discuss the nature of the exam with their patients, especially with high risk patients. OCS should become a part of each routine physical exam given by a primary care provider. It is recommended that medical schools include OCS in their curriculum for medical providers such as doctors, nurses, and physician's assistants. Routine dental care needs to be made available to lowincome and uninsured adults. More importantly, in addition to OCS during routine office visits, dental and medical offices should offer assistance in the reduction of risk factors for oral cancer, particularly in referral to tobacco-use cessation programs [13].

\section{Lung Cancer}

Lungs are in direct contact with the environmental air. About $10,000 \mathrm{~L}$ of air per day are transported through the airways to the lungs during breathing. A variety of pollutants, particles, bacteria, and viruses can be deposited into the airways. The respiratory epithelium is considered the first line of defense which forms a continuous lining to the airways. This lining has a protective physical and functional barrier to external harmful agents [20].

Lung cancer accounts for $13 \%$ (1.6 million) of the total cancer cases and 18\% (1.4 million) of the deaths in 2008 . Lung cancer was the most commonly diagnosed malignancy and a leading cause of cancer death in males in 2008 globally. In females, it was the fourth most commonly diagnosed cancer and the second leading cause of cancer death [10].

In males, the highest lung cancer incidence rates are in Eastern and Southern Europe, North America, Micronesia \& Polynesia, and Eastern Asia, while the lowest rates are in sub-Saharan Africa [10].

The estimated new cases of lung and bronchus cancers in the United States in 2009 was 116,090 males $(15 \%)$ and 103,350 females (14\%). The estimated deaths from lung and bronchus cancers in the United States in 2009 was 88,900 males $(30 \%)$ and 70,490 females $(26 \%)$. The risk of developing lung cancer is about 23 times higher in male smokers and 13 times higher in female smokers, compared to lifelong nonsmokers [4].

Cigarette smoking contributes to premature death and is the leading cause of preventable mortality and morbidity in the United States. Smoking related diseases cause more death than alcohol, drug use, homicide, and suicide combined. It was reported that most lung cancer deaths are attributable to cigarette smoking. Routine mortality statistics confirmed the link of smoking to lung cancer. The results that are obtained from case control and cohort studies using epidemiologic study designs to evaluate exposure/disease association causally linked smoking to lung cancer [21].

At the end of the 20th century, lung cancer had become one of the leading causes of preventable death. The biological studying of respiratory carcinogenesis and epidemiologic evidence has supported the conclusion that smoking causes lung cancer [22]. 
Despite the fact that tobacco had been widely used throughout the world for centuries, the pandemic of lung cancer increased after the introduction of manufactured cigarettes with addictive properties, which resulted in a continuous exposure of the lung to inhaled carcinogens. The involuntary inhalation of tobacco smoke by nonsmokers, known as passive smoking, can also leads lung cancer [23].

Chronic exposure to tobacco smoke is the major risk factor for development of lung cancer. Tumor promotion is due to induction and stimulation of inflammation that results in enhanced pneumocyte proliferation. Compared with never smokers, smokers with no attempts to quit are approximately 20-fold higher risk to lung cancer. Age, number and duration of cigarettes per day can increase the risk for lung cancer among cigarette smokers. This observation has been made repeatedly in cohort and case-control studies. Duration of smoking causes more risk to develop lung cancer than the amount smoked per day. Thus, a tripling of the number of cigarettes smoked per day was estimated to triple the risk, whereas a tripling of duration of smoking was estimated to increase the risk 100-fold. However, in general, studies have shown comparable reductions in risk after cessation regardless of gender, type of tobacco smoked, and histologic type of lung cancer [24].

A current research approach, termed molecular epidemiology, relates the population and laboratory tools that are used to address susceptibility to environmental carcinogens. Molecular epidemiology characterizes the sequence of molecular and cellular changes as a nonmalignant cell becomes malignant and genetic factors that affect the susceptibility to tobacco smoke $[25,26]$. There is sufficient epidemiological evidence on the association between tobacco smoking and cancer since the 1950s. In 1957, a study group examined the scientific evidence on the effects of tobacco smoking on health and concluded that "the sum total of scientific evidence establishes beyond reasonable doubt that cigarette smoking is a causative factor in the rapidly increasing incidence of human epidermoid carcinoma of the lung" [23].

Considering the link between active smoking and lung cancer, the relationship of environmental tobacco smoke (ETS) and lung cancer risk has been widely studied.

ETS is a mixture of sidestream and mainstream smoke produced by the burning of tobacco products (cigarettes, cigars or pipes) exhaled by the smoker. The diluted sidestream smoke which is the main component of ETS still represents a source of the same toxic substances and carcinogens that are inhaled by the active smoker but with different proportions. Although the quantity of carcinogens from ETS is far less than from smoking, urinary metabolites of tobacco specific carcinogens such as NNK are detectable in nonsmokers exposed to ETS. This biological evidence supports the role of ETS in lung cancer development.

To date, many epidemiological studies have been conducted to evaluate the effect of ETS exposure and lung cancer risk, mostly by comparing spouses of smokers and never smokers. Analyses of these data confirmed that spousal and workplace exposure to ETS is associated with a $20-25 \%$ increased risk of lung cancer [27].

Based on this data, the US National Institutes of Health (NIH) and International Agency for Research in Cancer (IARC) have officially designated ETS as a human carcinogen. Nonetheless, the overall evidence indicates that ETS is a relatively weak carcinogen and most lung cancers in never smokers cannot be explained by ETS exposure alone [28].

Cigarette smoke contains 50 known as carcinogenic compounds which induce lung cancers in laboratory animals. Among those tobacco-specific carcinogens (TSCs) is the Nicotine-derived nitrosoaminoketone (NNK). It is one of the most abundant nitrosamines in the human environment and the most potent specific carcinogen for lung tissues [20].

Other carcinogens that are present in tobacco smoke and strongly associated with lung cancer development are polycyclic aromatic hydrocarbons and nicotine derived nitrosaminoketone, which leads to genetic mutations through DNA adduct formation. The human lung epithelium has tobacco-specific carcinogens receptors, transporters, and ion channels in the airway which may have a role in this biological defense against tobacco smoke. Multidrug resistance protein-2 (MRP2) and cystic fibrosis transmembrane conductance regulator (CFTR), [two ATPbinding cassette $(\mathrm{ABC})$ transporters], are localized to the apical surfaces of plasma membrane in polarized lung epithelial cells. Although the causes of lung cancer are mostly environmental (smoking, pollution, etc.), there is an individual variation in susceptibility to respiratory carcinogens. The risk for the disease can rise if there are combined effects between exposure to the etiologic agents and the individual susceptibility to these agents [29].

The environment plays an important role in relation to epigenetics. The reversible nature of epigenetics states that an organism is allowed to respond to the changes in the environment. For example exposure to ultraviolet radiation, toxic compounds, and diet change. Ultraviolet radiation was identified as an environmental carcinogen with "epigenotoxic" effects. Lung cancer has a set of important risk factors and has well recognized synergistic interactions between these risk factors. Estimates for lung cancer indicate that in the United States, active smoking is responsible for $90 \%$ of lung cancer cases [30].

The histological findings of lung cancer have confirmed four major types of lung cancer and several minor or rare forms. Based on clinical pathological features, lung cancer can be divided into two broad categories of small cell lung cancer (SCLC) and non-small-cell lung cancer (NSCLC). NSCLCs are further divided into three major types, squamous cell carcinoma (SCC), adenocarcinoma (ADC) and large cell carcinomas. Bronchioloalveolar carcinomas make up a small component of NSCLCs.

All major histological types of lung cancer are associated with smoking, although the association is stronger for SCLC and for SCC than for NSCLCs [31]. The major histological types of lung cancer SCLC, SCC and ADC may originate 
from different compartments in the lung. SCLC and SCC and some $(20 \%)$ ADCs are thought to arise in the central compartment of conducting bronchial airways from a putative stem cell, the basal bronchial cell. These stem cells are capable of differentiation into both ciliated and mucous cells, the later believed to form ADCs [32].

The terminal respiratory unit, consisting of the peripheral compartment of respiratory bronchioles and alveoli, gives rise to peripheral ADC. It is believed that stem cells for selfrenewal and proliferation are the bronchioloalveolar stem cells. Which are also known as Clara cells (expressing Clara Cell kD protein (CC10) and type-II Pneumonocytes (expressing surfactants and their transcription factor, TTF1) [33].

Lung cancer in non-smokers or never-smokers usually arises in the peripheral compartment, driven by poorly identified exogenous carcinogens, among which passive smoking should be considered. The majority of lung cancers, $85 \%$ of NSCLC and $98 \%$ of SCLC, arise in smokers [33]. This change of histological pattern has occurred over a short period of time; a few decades which was hypothesized to be due to the widespread consumption of cigarettes with lowered tar and nicotine contents in many countries leading to changes in the anatomic location and histological type of lung cancer [34].

Tobacco associated carcinogens appear to target both central and peripheral airway compartments. Nicotinederived nitrosoaminoketone (NNK) is a potent tobaccospecific carcinogen that directly induces mutation-associated adenocarcinoma in rodents [35].

\section{Cancer Prevention, Tobacco Control, and Lung Cancer}

Preventive measures that delay the age of onset of smoking in a population could have significant impact on the incidence of lung cancer by shortening the duration of smoking. As the period of abstinence from smoking cigarettes increases, the risk for lung cancer decreases. Nevertheless, even for periods of abstinence of 40 years, the risk for lung cancer among former smokers remains elevated compared with never-smokers. The best preventive measure for lung cancer is, not starting to smoke followed by early elimination (quitting) of smoking which decreases the mortality of this disease. However, there is still risk of lung cancer developing in non-smokers because of other reasons. So, even if everyone stopped smoking today, lung cancer incidence and mortality would continue to be high for several decades. Family history is considered as a high risk factor in the development of lung cancer [34].

Early smoking cessation can reduce the risk to develop primary tumor of all major histological types of lung cancer to a great extent, especially small cell and squamous cell tumors. Cancer related morbidity and mortality could be potentially reduced as a result of quitting smoking after diagnosis but in the absence of previous data from randomized controlled trials no causal inferences can be made [27].
According to US Surgeon General's Report, the benefits of smoking cessation can be outlined as following: regardless of age, smokers live shorter lives than people who quit. Quitting before the age of 50 cuts the risk of dying within 15 years in half in comparison with those who continue to smoke. Quitting smoking significantly decreases the risk of lung, laryngeal, esophageal, oral, pancreatic, bladder, and cervical cancers [36].

Also, epigenetics plays a role in cancer prevention. Epigenetics are referred to as heritable changes in DNA methylation and chromatin structure. These changes are potentially reversible and are responsible for the initiation and development of malignant changes that are known as the carcinogenesis process [36]. These epigenetic modifications are promising new targets for cancer prevention trials.

After reports and publications about smoking, cigarette smoking had decreased by about $15 \%$ with a steady decline of about $5 \%$ over a period of several months. Also, the steady stream of other public health interventions such as the ban of broadcast advertising for cigarettes and doubling of the federal cigarette tax have been associated with a steady decline in smoking rates [37].

Tobacco control policy will continue to be an important issue in the United States and other parts of the world. The Framework Convention on Tobacco Control concurs many of the basic principles provided by public health advocates and scientists concerning the harmful effect of tobacco and reduction strategies [38].

\section{Genetic Susceptibility and Genome Wide Association Studies of Oral and Pharyngeal Cancers}

The continuous advancement in genotyping technology has enriched genetic association studies. Hundreds of thousands of variants were genotyped across the entire genome and these studies detected many highly statistically significant variants in the human chromosome 8q24 region that were associated with oral and pharyngeal cancer [39].

The $8 \mathrm{q} 24$ region has been of accumulating research interest in cancer development and epidemiology. Amplification within the $8 \mathrm{q} 24$ loci has been observed within a diverse group of cancers. Recent genome-wide association (GWA) studies identified associations between genetic variants or single nucleotide polymorphisms (SNPs): DG8S737, rs1447295, rs16901979, and rs6983267, along the 8 q24 region and oral and pharyngeal cancer among multiple study populations. Many studies suggest that $8 \mathrm{q} 24$ is associated with tobacco smoking-related cancer sites (lung, oropharynx, larynx, esophagus, stomach, liver, bladder, and pilot studies on nasopharynx and kidney) [40].

Many meta-analysis studies on the genetics of oral cancer have been published, and many positive results have been identified; however, there might be some false-positive results. A total of 14 variants were recognized from metaanalyses and pooled analyses. Among these 14 variants, 9 variants were reported to be significantly associated with the 
risk of oral cancer (CYP1A1-MspI, CYP2E1-RsaI/PstI, MTHFR-C677T, p73-G4C14-to-A4T14, XRCC1-Arg194Trp, CYP1A1-Ile462Val, GSTM1- \pm , and NAT2 slow vs rapid) $[41,42]$.

\section{Genetic Susceptibility and Genome Wide Association Studies of lung Cancers}

Understanding of both genetic susceptibility and epigenetic mechanisms related to the disease initiation and progression can add valuable information to achieve accurate diagnosis, better clinical management, and prognosis for lung cancer.

DNA hypermethylation is the mechanism responsible for inactivation of tumor suppressor gene that underlies the tumor-specific biological profile and the biomarkers related to the early detection of various types of cancer. Alteration of DNA methylation is a common feature for human cancer, leading to the epigenetic disturbance that is most likely responsible for the changed patterns of the gene expression and the different phenotypes of cancer cells. The hypermethylated state negatively encodes the proteins that regulate the cell proliferation and maintain the genome stability as a tumor suppressor and DNA-repairing mediators which are linked with the transcriptional silencing state of their expression in cancer cells [43-45].

DNA methylation can be used as a biomarker for cancer detection especially in the early stage of the disease. The environmental factors have a significant influence on both carcinogenesis and the clinical features of the disease through the epigenetic makeup of the cell [46].

Carcinogens found in cigarettes represent the major risk factors associated with lung cancer. Nicotine and its metabolites have the ability to activate neuronal nicotinic acetylcholine receptors (nAChR) which have a role in cancer signaling pathways associated with cell proliferation, apoptotic inhibition, and angiogenesis. Earlier studies have implicated the link between nAChRs and these pathways particularly the a7 nAChR. The recent genetic studies suggest that other subtypes should be investigated in future studies [47].

Several studies showed that the $15 \mathrm{q} 25$ is statistically associated with the smoking habit, smoking-related chronic obstructive lung diseases (COPD) and lung cancer [48]. The 7 receptor subunit is vital for nicotine-mediated cell proliferation and Src function is indispensable for nicotine to induce proliferation and angiogenesis. Also, it is expected that muscle-type nAChR subunits have a role in proliferation. Three different studies from Europe showed a susceptibility locus which maps to nicotinic acetylcholine receptor subunit genes on chromosome15q24-25 [49-51]. Genes for a3, a5 and b4 subunits are contained in this locus. This locus has many variations in smoking-related lung cancer and peripheral arterial diseases. These studies showed that there may be a direct correlation between the status and function of nAChRs and the development and progression of lung cancer in smokers. Also, the non small cell lung cancers (NSCLCs) from never smokers express higher levels of a6b3 subunits whereas smokers show higher expression of a1, a3 and a7 subunits and a lower expression of a6b3 nAChR subunits. Nicotine may stimulate nAChR which contributes to the progression of lung cancers. Other signaling molecules can be activated by nAChR stimulation. These include activation of Src kinase cascade, PI3-Akt pathway, ERK/MAP kinase cascade, NFkB pathway and cyclic AMP signaling cascade [47].

Genome-wide association (GWA) studies have found a linkage between the variation on the long arm of chromosome 15 (15q24-25.1) and lung cancer risk. That variation in this region may modify individual susceptibility to lung and pancreatic cancer [52].

Although nicotine is known to have limited tumorigenicity, it can promote cell proliferation and angiogenesis. These characteristics of nicotine may facilitate the growth of tumors that are already developed. Another study showed that nicotine can significantly promote the progression and metastasis of tumors in mouse models of lung cancer via nicotine intraperitoneal injections or through transdermal patches. The results indicated that nicotine could induce invasion and epithelial-mesenchymal transition (EMT) in cultured lung, breast and pancreatic cancer cells. This study demonstrated that administration of nicotine can promote tumor growth and metastasis in immunocompetent mice. They suggested that nicotine can facilitate the progression and metastasis of tumors that was originally developed by tobacco carcinogens [53].

Identification of susceptibility genes could play a great role in personalized prediction, risk estimation, and individualized therapy for lung cancer. Increased familial risk of lung cancer is a noticeable indication of a genetic contribution but still not a sufficient one as familial clustering of non-genetic risk factors chiefly cigarette smoking can have an influence too. Also, it was estimated that the familial risk of lung cancer between offspring would be expected to increase by about $20 \%$ with increased familial tendency to smoke. However, other factors, including genetic susceptibility, are the main contributors to this increased risk [53].

Genes that may affect lung cancer are classified into three groups: rare high-risk variants (risk of 10 or higher and prevalence of $1 \%$ or less), moderate-risk variants (risk of around $2-5$ and prevalence of not more than 5\%), and common low-risk variants (risk of between 1.1- 1.5 and prevalence of more than 5\%).

Most genetic risk is likely to involve several genes of moderate and low risk. Although identification of gene susceptibility and lung cancer has confirmed the precise effect of the genetic relation in lung cancer functionally, it is still unknown and more confirmation studies are needed. A key cell-cycle control gene, named I157T variant of CHEK2, that activates cell cycle checkpoints in response to DNA damage was associated with lung cancer. It has a main role maintaining the genetic integrity. Understanding of this association will be important in order to develop CHEK2based therapeutics [54-56]. 
Studies have shown associations between markers on chromosome $6 \mathrm{q}$ to lung cancer, chronic obstructive pulmonary diseases (COPD) and lung function together with overlap in candidate genes. Therefore, future research into underlying genetic mechanisms of lung disease in relation to family history and lung function data looks promising. The identification of genes for lung cancer and COPD will require larger linkage and association studies that would benefit from the collection of lung function and lung disease history with detailed family histories [55].

Genome-wide association (GWA) studies use genotyping of up to one million genetic variants but do not refer to the functional significance of the individual variants studied. GWA studies provide partial coverage of common variants and limited coverage of rarer variants. In 2009, GWA studies identified more than 80 common variants indirectly linked with different cancer sites [57].

GWA studies showed a link between 8q24 variants, particularly rs6983267 and cancer sites, particularly in upper aero-digestive tract (UADT) cancers and lung cancer among smokers. These associations between rs6983267 and upper aero-digestive tract cancers were analyzed by genotypes. The GG genotype was strongly associated with the UADT cancer with evidence of dose related response relationship between rs6983267 and the UADT cancers when stratified by tobacco smoking [40].

The genetic region $15 \mathrm{q} 25$ is a highly susceptible region. It contains six identified coding regions. Two of the GWA studies recognized variants by their direct association with lung cancer risk but the third study identified an association with the same genetic region and smoking quantity, and concluded that the variant increases lung cancer risk by smoking. Peripheral arterial and chronic obstructive pulmonary diseases are linked to the variant allele [58].

Although the association between $15 \mathrm{q} 25$ variants and smoking intensity is repeatedly identified (about $80 \%$ increase in lung-cancer risk for individuals who inherit two risk alleles), this alone is not enough evidence to strongly link the association with lung cancer. This is based on evidence from studies of non-smokers and lung cancer. A Japanese study of the same $15 \mathrm{q} 25$ variants that increases the risk in European populations found that these variants were associated with lung-cancer risk to a similar extent among never and ever smokers [58].

Lung cancer prognosis is significantly low due to the high metastatic potential and limited response to treatment. Comprehensive genetic studies of interindividual variation may influence the response to chemotherapy and overall survival of treated patients.

Genome-wide association studies of chemotherapeutic agents as cisplatin cytotoxicity in lymphoblastoid cell lines have also been performed and identified several novel associations between genetic variation and cytotoxicity. The variants recognized were located in genes not previously considered as candidates for chemotherapy response [52, 59].
Adjusted DNA methylation is the normal condition that exists in all normal tissues and represents the gene expression patterns in each cell type at any developmental stage. However, in malignant tissues the scenario is different. It was documented over the last 20 years that the level of 5-methylcytosine declines in malignant tissues of several tumor-types. DNA hypomethylation usually happens due to loss of methylation in repetitive sequences such as ribosomal DNA repeats, satellite or centromeric repeats which are heavily methylated in normal tissues. Loss of DNA methylation mechanisms are still under investigation and not completely understood [60].

Hypomethylation of repeat sequences corresponds with chromatin changes that in turn cause genomic instability, a feature of cancer genomes, and could even be the stimulator for the tumorigenesis process [61]. Also, there is another mechanism where epigenetic alterations play a role in carcinogenesis, known as hypermethylation, which leads to gene silencing. Epigenetic silencing of a tumor suppressor gene results in loss of function similar to a genetic deletion or mutation. Because epigenetic alterations do not alter the genetic sequence and are not permanent, this process is reversible and the possibility for gene reactivation is possible.

Tumor suppressor genes identified in cancer have been evaluated for epigenetic alterations in tumors that do not show a gene mutation. The list of such epigenetically silenced genes is extensive and includes genes with functions in cell cycle regulation $[62,63]$.

In lung cancer, p16INK4a methylation was detected at the earliest stages and increases with disease progression. It was also detected in normal bronchial epithelium from current and former smokers, and in sputum from high risk individuals and cancer patients. However, p16 methylation might not be predictive for cancer development, but rather facilitates acquisition of additional genetic and/or epigenetic alterations [64].

These epigenetic alterations are now under investigation to be used as biomarkers for early detection of cancer. They may be potential targets for chemopreventive therapy [65]. To detect these biomarkers, polymerase chain reaction (PCR)-based assays are used to detect cancer-specific methylation events in body fluids such as sputum, urine or plasma. Recently, DNA methylation profiling has developed to assess the level of the epigenetically silenced genes in the order of thousands of genes, in a cancer genome [65, 66].

Over the last few years, evidence has accumulated that chemopreventive agents have an influence on DNA methylation and chromatin remodeling [61]. Several studies at the cell culture level, have proved that chemopreventive agents can be used effectively to target the epigenome by several interacting pathways. These agents (micronutrients, vitamins, butyrate, polyphenols from various sources, selenium, sulfur-containing compounds, other bioactive dietary components, antibiotics, and pharmacological agents) have potential to regulate gene expression by epigenetic mechanisms [67]. 
They affect DNA methylation, tumor suppressor genes silencing, histone modifications, and miRNAs expression during carcinogenesis. These effects have a potential impact on mechanisms relevant for chemoprevention, including signal transduction mediated by nuclear receptors and transcription factors, cell cycle progression, cellular differentiation, apoptosis induction, senescence, and others. Further investigations at the animal level are needed to show that these observations are functionally linked in order to reveal the real chemopreventive efficacy pathway and whether it is mediated by epigenetic gene regulation, is based on other chemopreventive mechanisms, or is a combination of both [68].

Tumor suppressor genes that are irreversibly inactivated by genetic alterations, genes silenced by epigenetic modifications, are still intact and can be reactivated by small molecules or dietary factors acting as modifiers of epigenetic mechanisms [56].

\section{CONCLUSION}

Although smoking is well-known, established risk factor linked to oral, pharyngeal and lung cancer, genetic susceptibility could be the key for the development of cancer in these sites. Every smoker has a higher chance of developing oral cancer, pharyngeal and lung cancers compared to a never smoker. As the period of abstinence from smoking cigarettes increases, the risk for lung cancer decreases. Recent studies showed high association between genetics and cancer. With evidence from new studies being reported, hypomethylation of repeat sequences corresponds with chromatin changes that in turn cause genomic instability, a feature of cancer genomes. DNA methylation mechanisms are still not completely understood. Epigenetic alterations are under investigation to be used as biomarkers for early detection of cancer. They may be potential targets for chemopreventive therapy. Epigenetic modifications are promising new targets for cancer prevention trials. Research on interactions of diet, DNA methylation, and their influence on cancer prevention should be encouraged. Statistically, the risk of developing lung cancer is about 23 times higher in male smokers and 13 times higher in female smokers, compared to lifelong nonsmokers. Therefore, it is important to reduce the amount of tobacco used throughout the world. We need to increase the awareness of the public not only about the harmful effects caused by tobacco use and the complications it may cause, but also increase the awareness of the role of genetics, diet, and consumption of alcohol. It is important to discuss the consequences of these cancers and other complications which arise from tobacco use to help spread the message of how dangerous tobacco can be.

\section{CONFLICT OF INTEREST}

The authors confirm that this article content has no conflict of interest.

\section{ACKNOWLEDGEMENTS}

Declared none.

\section{REFERENCES}

[1] American Cancer Society, Cancer Facts and Figures http://wwwcancerorg/research/cancerfactsstatistics/cancerfactsfigur es2014/. 2014: pp. 43-5.

[2] Gandini S, Botteri E, Iodice S, et al. Tobacco smoking and cancer: a meta-analysis. Int J Cancer 2008; 122(1): 155-64.

[3] van der Waal I. Are we able to reduce the mortality and morbidity of oral cancer; some considerations. Med Oral Patol Oral Cir Bucal 2013; 18(1): e33-7.

[4] Cancer Facts \& Figures 2012. Atlanta: American Cancer Society 2012.

[5] Rodu B, Jansson C. Smokeless tobacco and oral cancer: a review of the risks and determinants. Crit Rev Oral Biol Med 2004; 15(5): 252-63.

[6] Vargas-Ferreira F, Nedel F, Etges A, Gomes AP, Furuse C, Tarquinio SB. Etiologic factors associated with oral squamous cell carcinoma in non-smokers and non-alcoholic drinkers: a brief approach. Braz Dent J 2012; 23(5): 586-90.

[7] Bhisey RA. Chemistry and toxicology of smokeless tobacco. Indian J Cancer 2012; 49(4): 364-72.

[8] Stepanov I, Yershova K, Carmella S, Upadhyaya P, Hecht SS Levels of (S)-N'-Nitrosonornicotine in U.S. Tobacco products. Nicotine Tob Res 2012; 15(7): 1305-10.

[9] Balbo S, James-Yi S, Johnson CS, et al. (S)-N'-Nitrosonornicotine, a constituent of smokeless tobacco, is a powerful oral cavity carcinogen in rats. Carcinogenesis 2013; 34(9): 2178-83.

[10] Jemal A, Bray F, Center MM, Ferlay J, Ward E, Forman D. Global cancer statistics. CA Cancer J Clin 2011; 61(2): 69-90.

[11] Petti S, Masood M, Scully C. The magnitude of tobacco smokingbetel quid chewing-alcohol drinking interaction effect on oral cancer in South-East Asia. A meta-analysis of observational studies. PLoS One 2013; 8(11): e78999.

[12] Agrawal M, Pandey S, Jain S, Maitin S. Oral cancer awareness of the general public in gorakhpur city, India. Asian Pac J Cancer Prev 2013; 13(10): 5195-9.

[13] Viswanath A, Kerns TJ, Sorkin JD, Dwyer DM, Groves C, Steinberger EK. Self-reported oral cancer screening by smoking status in Maryland: trends over time. J Public Health Dent 2013; 73(4): 261-70.

[14] Varela-Lema L, Ruano-Ravina A, Juiz-Crespo MA, Barros-Dios JM. Tobacco consumption and oral and pharyngeal cancer in a Spanish male population. Cancer Lett 2010; 288(1): 28-35.

[15] Zhou J, Michaud DS, Langevin SM, McClean MD, Eliot M, Kelsey KT. Smokeless tobacco and risk of head and neck cancer: evidence from a case-control study in new England. Int J Cancer 2013; 132(8): 1911-7.

[16] Warnakulasuriya S. Causes of oral cancer--an appraisal of controversies. Br Dent J 2009; 207(10): 471-5.

[17] Lubin JH, Muscat J, Gaudet MM, et al. An examination of male and female odds ratios by BMI, cigarette smoking, and alcohol consumption for cancers of the oral cavity, pharynx, and larynx in pooled data from 15 case-control studies. Cancer Causes Control 2011;22(9): 1217-31.

[18] D'Souza G, Kreimer AR, Viscidi R, et al. Case-control study of human papillomavirus and oropharyngeal cancer. N Engl J Med 2007; 356(19): 1944-56

[19] Ang KK, Harris J, Wheeler R, et al. Human papillomavirus and survival of patients with oropharyngeal cancer. N Engl J Med 2010; 363(1): 24-35

[20] Li C, Schuetz JD, Naren AP. Tobacco carcinogen NNK transporter MRP2 regulates CFTR function in lung epithelia: implications for lung cancer. Cancer Lett 2010; 292(2): 246-53.

[21] White C. Research on smoking and lung cancer: a landmark in the history of chronic disease epidemiology. Yale J Biol Med 1990; 63(1): 29-46.

[22] Rosen G. A History of Public Health. Expanded ed. Baltimore: Johns Hopkins University Press 1993.

[23] Smoking and Health; joint report of the Study Group on Smoking and Health. Science 1957; 125(3258): 1129-33.

[24] Takahashi H, Ogata H, Nishigaki R, Broide DH, Karin M. Tobacco smoke promotes lung tumorigenesis by triggering IKKbeta- and JNK1-dependent inflammation. Cancer Cell 2010; 17(1): 89-97. 
[25] Cornfield J, Haenszel W, Hammond EC, Lilienfeld AM, Shimkin MB, Wynder EL. Smoking and Lung Cancer: Recent Evidence and a Discussion of Some Questions. Int J Epidemiol 2009; 38(5): 1175-91.

[26] Alberg AJ, Samet JM. Epidemiology of lung cancer. Chest 2003; 123(1 Suppl): 21S-49S.

[27] The Health Consequences of Involuntary Exposure to Tobacco Smoke: A Report of the Surgeon General. Rockville, MD: U.S. Dept. of Health and Human Services, Public Health Service, Office of the Surgeraleon Gen 2006.

[28] Sun S, Schiller JH, Gazdar AF. Lung Cancer in never smokers - a different disease. Nat Rev Cancer 2007; 7(10): 778-90.

[29] Cantin AM, Hanrahan JW, Bilodeau G, et al. Cystic fibrosis transmembrane conductance regulator function is suppressed in cigarette smokers. Am J Respir Crit Care Med 2006; 173(10): 1139-44.

[30] Peto R, Lopez AD, Boreham J, Thun M, Heath Jr C. Mortality from Smoking in Developed Countries 1950-2000: Indirect Estimation From National Vital Statistics. Lancet: Oxford University Press 1994.

[31] Brambilla E, Travis WD, Colby TV, Corrin B, Shimosato Y. The new World Health Organization classification of lung tumours. Eur Respir J 2001; 18(6): 1059-68.

[32] Weinstein IB, Joe A. Oncogene addiction. Cancer Res 2008; 68(9): 3077-80; discussion 80.

[33] Brambilla E, Gazdar A. Pathogenesis of lung cancer signalling pathways: roadmap for therapies. Eur Respir J 2009; 33(6): 148597.

[34] Khuder SA. Effect of cigarette smoking on major histological types of lung cancer: a meta-analysis. Lung Cancer 2001; 31(2-3): 13948.

[35] Hecht SS. Cigarette smoking and lung cancer: chemical mechanisms and approaches to prevention. Lancet Oncol 2002; 3(8):461-9.

[36] Bach PB. Smoking as a factor in causing lung cancer. JAMA 2009; 301(5): 539-41.

[37] Jemal A, Thun MJ, Ries LA, et al. Annual report to the nation on the status of cancer, 1975-2005, featuring trends in lung cancer, tobacco use, and tobacco control. J Natl Cancer Inst 2008; 100(23): 1672-94.

[38] CancerStats: Cancer Statistics for the UK : Cancer Research UK 2007; Available from: http://www.cancerresearchuk.org/cancerinfo/cancerstats/

[39] Dong LM, Potter JD, White E, Ulrich CM, Cardon LR, Peters U. Genetic susceptibility to cancer: the role of polymorphisms in candidate genes. JAMA 2008; 299(20): 2423-36.

[40] Park SL, Chang SC, Cai L, et al. Associations between variants of the 8q24 chromosome and nine smoking-related cancer sites. Cancer Epidemiol Biomarkers Prev 2008; 17(11): 3193-202.

[41] Liao G, Wang Y, Zhou YQ, et al. Host genetic susceptibility to oral cancer: evidence from meta-analyses and pooled analyses. Oral Dis 2014; 20(7): 644-9.

[42] Chang CQ, Yesupriya A, Rowell JL, et al. A systematic review of cancer GWAS and candidate gene meta-analyses reveals limited overlap but similar effect sizes. Eur J Hum Genet 2014; 22(3): 4028 .

[43] Esteller M. Epigenetics in cancer. N Engl J Med 2008; 358(11): 1148-59.

[44] Zhu J, Yao X. Use of DNA methylation for cancer detection: promises and challenges. Int J Biochem Cell Biol 2009; 41(1): 14754.

[45] Lin Q, Geng J, Ma K, et al. RASSF1A, APC, ESR1, ABCB1 and HOXC9, but not p16INK4A, DAPK1, PTEN and MT1G genes were frequently methylated in the stage I non-small cell lung cancer in China. J Cancer Res Clin Oncol 2009; 135(12): 1675-84.
[46] Improgo MR, Scofield MD, Tapper AR, Gardner PD. From smoking to lung cancer: the chrna5/a3/b4 connection. Oncogene 2010; 29(35): 4874-84.

[47] Davis R, Rizwani W, Banerjee S, et al. Nicotine promotes tumor growth and metastasis in mouse models of lung cancer. PLoS One 2009; 4(10): e7524.

[48] Galvan A, Dragani TA. Nicotine dependence may link the $15 q 25$ locus to lung cancer risk. Carcinogenesis 2010; 31(3): 331-3.

[49] Amos CI, Wu X, Broderick P, et al. Genome-wide association scan of tag SNPs identifies a susceptibility locus for lung cancer at 15q25.1. Nat Genet 2008; 40(5): 616-22.

[50] Hung RJ, McKay JD, Gaborieau V, et al. A susceptibility locus for lung cancer maps to nicotinic acetylcholine receptor subunit genes on 15q25. Nature 2008; 452(7187): 633-7.

[51] Thorgeirsson TE, Geller F, Sulem P, et al. A variant associated with nicotine dependence, lung cancer and peripheral arterial disease. Nature 2008; 452(7187): 638-42.

[52] Chen J, Wu X, Pande M, et al. Susceptibility locus for lung cancer at $15 q 25.1$ is not associated with risk of pancreatic cancer. Pancreas 2011; 40(6): 872-5.

[53] Bermejo LJ, Hemminki K. Familial lung cancer and aggregation of smoking habits: a simulation of the effect of shared environmental factors on the familial risk of cancer. Cancer Epidemiol Biomarkers Prev 2005; 14(7): 1738-40.

[54] Wu X, Gwyn K, Amos CI, Makan N, Hong WK, Spitz MR. The association of microsomal epoxide hydrolase polymorphisms and lung cancer risk in African-Americans and Mexican-Americans. Carcinogenesis 2001; 22(6): 923-8.

[55] Park JY, Chen L, Elahi A, Lazarus P, Tockman MS. Genetic analysis of microsomal epoxide hydrolase gene and its association with lung cancer risk. Eur J Cancer Prev 2005; 14(3): 223-30.

[56] Schwartz AG, Ruckdeschel JC. Familial lung cancer: genetic susceptibility and relationship to chronic obstructive pulmonary disease. Am J Respir Crit Care Med 2006; 173(1): 16-22.

[57] Lips EH, Gaborieau V, McKay JD, et al. Association between a 15 q25 gene variant, smoking quantity and tobacco-related cancers among 17,000 individuals. Int J Epidemiol 2010; 39(2): 563-77.

[58] Shiraishi K, Kohno T, Kunitoh H, et al. Contribution of nicotine acetylcholine receptor polymorphisms to lung cancer risk in a smoking-independent manner in the Japanese. Carcinogenesis 2009; 30(1): 65-70.

[59] Plass C, Smiraglia DJ. Genome-wide analysis of DNA methylation changes in human malignancies. Curr Top Microbiol Immunol 2006; 310: 179-98.

[60] Gaudet F, Hodgson JG, Eden A, et al. Induction of tumors in mice by genomic hypomethylation. Science 2003; 300(5618): 489-92.

[61] Kopelovich L, Crowell JA, Fay JR. The epigenome as a target for cancer chemoprevention. J Natl Cancer Inst 2003; 95(23): 1747-57.

[62] Widschwendter M, Jones PA. DNA methylation and breast carcinogenesis. Oncogene 2002; 21(35): 5462-82.

[63] Risch A, Plass C. Lung cancer epigenetics and genetics. Int J Cancer 2008; 123(1): 1-7.

[64] Figueroa ME, Lugthart S, Li Y, et al. DNA methylation signatures identify biologically distinct subtypes in acute myeloid leukemia. Cancer Cell 2010; 17(1): 13-27.

[65] Lister R, Pelizzola M, Dowen RH, et al. Human DNA methylomes at base resolution show widespread epigenomic differences. Nature 2009; 462(7271): 315-22.

[66] Verma M, Maruvada P, Srivastava S. Epigenetics and cancer. Crit Rev Clin Lab Sci 2004; 41(5-6): 585-607.

[67] Fay JR, Crowell JA, Kopelovich L. Targeting epigenetic regulatory mechanisms in cancer chemoprevention. Expert Opin Ther Targets 2005; 9(2): 315-28.

[68] Ross SA, Dwyer J, Umar A, et al. Introduction: diet, epigenetic events and cancer prevention. Nutr Rev 2008; 66 (Suppl 1): S1-6. 